\title{
Monte-Carlo Simulation of the Impact of LED Power Uncertainty on Visible Light Positioning Accuracy
}

\author{
David Plets*, Sander Bastiaens*, Nobby Stevens ${ }^{\dagger}$, Luc Martens*, Wout Joseph* \\ ${ }^{*}$ Ghent University/imec, Dept. of Information Technology, Technologiepark 15, B-9052 Ghent, Belgium \\ ${ }^{\dagger}$ KULeuven-DraMCo, Gebroeders De Smetstraat 1, B-9000 Ghent, Belgium \\ david.plets@ugent.be
}

\begin{abstract}
This paper presents a simulation study of the impact of Light Emitting Diode (LED) output power uncertainty on the accuracy of Received Signal Strength (RSS)-based Visible Light Positioning (VLP). The actual emitted power of a LED is never exactly equal to the value that is tabulated in the datasheet, with possible variations (or tolerances) up to $20 \%$. Since RSS-based VLP builds on converting estimated channel attenuations to distances and locations, this uncertainty will impact VLP accuracy in real-life setups. A typical configuration with four LEDs is assumed here, and a Monte-Carlo simulation is executed to investigate the distribution of the resulting positioning errors for four tolerance values at seven locations. It is shown that median errors are the highest just below the LEDs. When tolerance values on the LED power increase from $5 \%$ to $20 \%$, median errors vary from at most $2 \mathrm{~cm}$ to at most $10 \mathrm{~cm}$. Maximal errors can be as high as $17 \mathrm{~cm}$ just below the LED, already for tolerance values of only $5 \%$, and increase up to $40 \mathrm{~cm}$ for tolerance values of $20 \%$.
\end{abstract}

\section{INTRODUCTION}

The introduction of Light Emitting Diodes (LEDs) has not only revolutionized the lighting world thanks to the increased energy efficiency and lifetime compared to traditional lighting bulbs, but it has also unlocked new applications. The ability to modulate the emitted light signal allows transmitting data, a technology known as Visible Light Communication (VLC) [1]. Another promising application of visible light is Visible Light Positioning (VLP) [2], [1], where e.g., the location of a photo diode (PD) is estimated. Advantages over well-known RadioFrequency (RF) solutions, such as Ultra-Wide-Band (UWB) time-of-arrival (ToA)-based positioning [3], Angle-of-Arrival (AoA)-based positioning, or Received Signal Strength Indicator (RSSI)-based location tracking [4], include achieving high positioning accuracies while maintaining a low deployment cost. Similarly to RF solutions, VLP can use AoA or RSSbased approaches. The latter approach is often preferred thanks to the lower complexity of the receiver. It builds on the link between observed light intensities and distances between the LED source and the receiving photo diode, using (or assuming) knowledge of the visible light channel attenuation as a function of distance. However, such conversion also requires exact knowledge of the transmitted power of each of the LEDs. In reality, the precision of these powers is limited, and uncertainties typically amount to around 5\% [5]. For Chips on Board (COBs) LEDs, tolerances are up to $\pm 10 \%$ [6], and for some LED manufacturers, uncertainties could be as high as $20 \%$. To the authors' knowledge, up to now, no research efforts have been made to characterize the impact of this uncertainty on the performance of VLP in terms of accuracy. Therefore, this paper will assess this impact for a simple VLP configuration by means of a Monte-Carlo simulation, in which it is assumed that each of the four deployed LEDs has a certain unknown (and uncorrelated) deviation on its assumed output power. This way, the cumulative distribution function (cdf) of the positioning error will be constructed for seven locations within the test site, and for four different tolerance values ( 5 , 10,15 , and $20 \%$ ).

Section II will present the visible light channel model that will be used in the positioning algorithm. In Section III, the simple VLP test setup that will be assumed for the simulations, will be discussed, while Section IV will present the positioning algorithm. Results will be discussed in Section V, and the main findings of this work will be summarized in Section VI.

\section{Channel Model}

In this work, only the Line-of-Sight (LoS) path between the transmitting LED source and the receiving PD will be assumed. Reflections are not considered as a source of "measurement noise", in order to be able to unambiguously assess the effect of the tolerance on the LEDs' output powers. For the same reason, no actual noise (e.g., shot noise or thermal noise) is initially considered in this study. The model parameters of the visible light channel are displayed in Fig. 1. The power $P_{R}$ received at the photo diode is calculated according to the channel model used in [7]:

$$
P_{R}=P_{E} \cdot h_{L o S},
$$

with $P_{E}$ the emitted optical power by the LED, which will thus be statistically distributed in this paper. $h_{L o S}$ is the channel gain along the direct link and can be described as follows:

$$
h_{L o S}=R_{E}(\phi, \gamma) \cdot \frac{A_{e f f}(\psi)}{d^{2}} \cdot T_{R}(\psi) \cdot G_{R}(\psi),
$$

where $R_{E}(\phi, \gamma)$ is the radiation pattern of the LED, which is axially symmetric in case of a Lambertian emitter and for order 


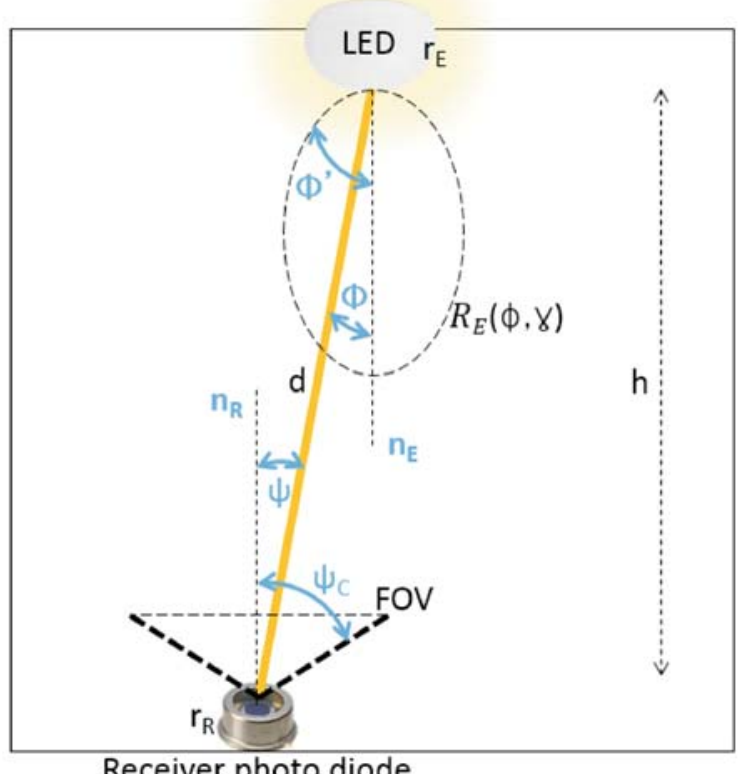

Receiver photo diode

Fig. 1: Overview of visible light channel.

$m$, reduces to $\frac{m+1}{2 \pi} \cos ^{m}(\phi)$, with $\phi$ the angle of irradiance (see Fig. 1). $T_{R}(\psi)$ and $G_{R}(\psi)$ are the optical filter's gain and the optical concentrator's gain at the receiver, respectively, with $\psi$ the angle of incidence. The field-of-view (FOV) of the photo diode is two times $\psi_{C}$ (see Fig. 1), such that $h_{L o S}$ becomes equal to zero for $|\psi|>\psi_{C}$. Within the FOV of the PD, $T_{R}(\psi)$ and $G_{R}(\psi)$ will be assumed equal to 1 in the following. $d$ is the distance between the LED and the PD, and $A_{e f f}$ is the effective photo diode area, which is equal to the photo diode area that is perpendicular to the angle of incidence $\psi$ :

$$
A_{e f f}(\psi)=A_{R} \cdot \cos (\psi),
$$

with $A_{R}$ the actual photo diode area, here equal to $1 \mathrm{~cm}^{2}$.

\section{Simulation CONFIGURATION}

The simulation tests will be executed for the room that is depicted in Fig. 2. The dimensions of the room are $5 \mathrm{~m} \times 5 \mathrm{~m}$, with a ceiling height of $2.5 \mathrm{~m}$. The receiver PD is assumed to be at a height of $0.85 \mathrm{~m}$. The Lambertian order $m$ of the LEDs is equal to 1 , for all four LEDs. In this scenario, we assume that the receiver height is fixed and known (e.g., a PD attached to the top of a cart), so the evaluation of the receiver location is reduced to a plane. A receiver grid of $2 \mathrm{~mm}$ will be considered here, meaning that the PD center can be located at $N_{L}=2500^{2}$ locations. Further, we assume that the receiver hardware is able to demultiplex the contributions of the different LED sources [8]. Four LEDs with an assumed optical power of $10 \mathrm{~W}$ are attached to the ceiling $(h=2.5 \mathrm{~m})$, at the locations indicated in Fig. 2. However, in reality, the emitted power $P_{E i}$ of $L E D_{i}(\mathrm{i}=1 . . \mathrm{N})$ will be normally distributed around the value of $10 \mathrm{~W}$ :

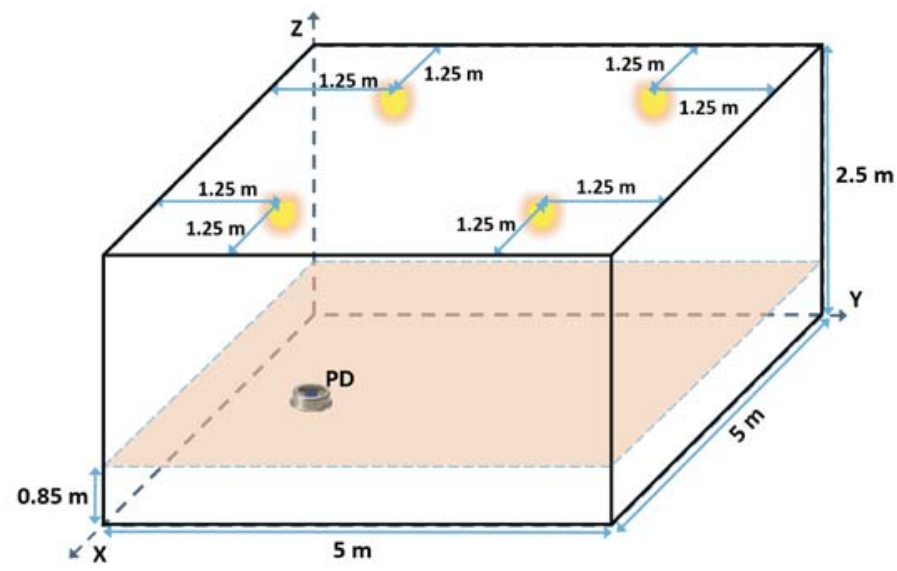

Fig. 2: Overview of the simulation setup.

$$
P_{E i} \sim \mathcal{N}\left(10, \sigma^{2}\right) .
$$

In this study, four tolerance values $T_{L E D}$ will be tested: $\pm 5 \%, \pm 10 \%, \pm 15 \%$, and $\pm 20 \%$. These tolerance values typically refer to 3-sigma deviations, meaning that the standard deviations $\sigma$ of our normal distributions around the $10 \mathrm{~W}$ value will correspond to $0.167 \mathrm{~W}, 0.333 \mathrm{~W}, 0.5 \mathrm{~W}$, and $0.666 \mathrm{~W}$, respectively. Further, it will be assumed that there is no correlation between the power values of the different LEDs in the room.

Fig. 3 shows the seven positions at which the location will be estimated. Thanks to the 8-fold symmetry of the setup, only locations within the triangle A-C-E need to be considered, since the cdfs of the positioning errors will repeat themselves at the corresponding locations of the different parts of the $5 \mathrm{~m} \times 5 \mathrm{~m}$ area. It should be noted though that each single simulation will result in an asymmetric setup, since for each simulation, the power of each LED will be randomly and independently chosen. However, the resulting cdf at corresponding locations will be the same when enough simulations are considered, due to the fact that the statistical distribution of the LED power is the same for each of the LEDs. Table I shows the coordinates of each of the positions A-G, where A is set at the center of the coordinate system, and $\mathrm{G}$ is the centroid of the triangle formed by the other points. In this work, a position estimation for each of the seven positions (A to $\mathrm{G}$ ) will be executed for 10000 random sets of LED power values $\left(P_{E 1}, P_{E 2}, P_{E 3}, \ldots, P_{E N}\right)$ in the MonteCarlo simulation $(N=4$ here. Each position estimation will be done according to the algorithm presented in Section IV.

\section{POSITIONING ALGORITHM}

The adopted positioning algorithms is based on the comparison of the set of so-called measured received photo diode powers $P_{R i}^{\text {meas }}$ from each $L E D_{i}(\mathrm{i}=1 \quad . . \mathrm{N})$ at the unknown PD location, with the set of fingerprinted PD powers $P_{R i}^{L, \text { model }}$ from $L E D_{i}$ at all $\left(2500^{2}\right)$ locations $\mathrm{L}$ in the grid. For the construction of the fingerprinting database of the 
TABLE I: Coordinates $(\mathrm{m})$ of the considered locations, where $\mathrm{A}$ is set at the center of the coordinate system, the $\mathrm{x}$-axis pointing rightwards, and the $\mathrm{y}$-axis pointing upwards (see Fig. 3).

\begin{tabular}{|r|r|r|r|r|}
\hline Location & $\mathbf{A}$ & $\mathbf{B}$ & $\mathbf{C}$ & $\mathbf{D}$ \\
\hline Coordinates & $(0,0)$ & $(1.25,1.25)$ & $(2.25,2.25)$ & $(2.25,1.25)$ \\
\hline \hline Location & $\mathbf{E}$ & $\mathbf{F}$ & $\mathbf{G}$ & \\
\hline Coordinates & $(2.25,0)$ & $(1.25,0)$ & $(1.67,0.83)$ & \\
\hline
\end{tabular}

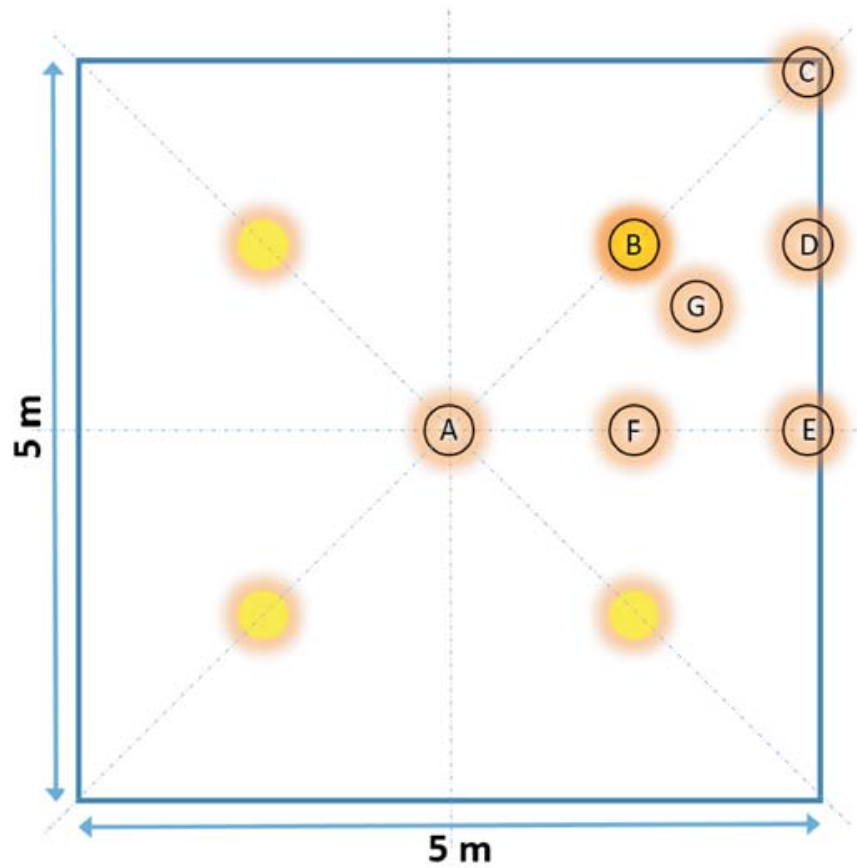

Fig. 3: Top view of the simulation setup, with indication of the four LEDs (yellow dots) and the seven locations (A to $G$ ) for which the positioning error will be evaluated.

$P_{R i}^{L, \text { model }}$ values, $P_{E}$ is assumed equal to $10 \mathrm{~W}$ for each LED, as it is the most probable value for $P_{E}$. The set of so-called measurements $\left(P_{R 1}^{\text {meas }}, P_{R 2}^{\text {meas }}, P_{R 3}^{\text {meas }}, \ldots, P_{R N}^{\text {meas }}\right)$ represent the observed values in the realistic setup investigated here. They are obtained from $\left(P_{E 1}, P_{E 2}, P_{E 3}, \ldots, P_{E N}\right)$, where $P_{E i}$ values are obtained as samples from $\mathcal{N}\left(10, \sigma^{2}\right)$. The larger the uncertainty on the $P_{E i}$ values (larger $\sigma^{2}$ values), the larger the positioning errors will be.

The algorithm estimates the unknown location $\mathrm{L}$ to be at the spot where the cost function $C_{\text {square }}^{L}$ has a minimum [9]:

$$
C_{\text {square }}^{L}=\sum_{i}^{N}\left(P_{R i}^{\text {meas }}-P_{R i}^{L, \text { model }}\right)^{2},
$$

Each position estimation thus consists of a comparison of the set of measurements $\left(P_{R 1}^{\text {meas }}, P_{R 2}^{\text {meas }}, P_{R 3}^{\text {meas }}, \ldots, P_{R N}^{\text {meas }}\right)$ against all $\left(P_{R 1}^{L, \text { model }}, P_{R 2}^{L, \text { model }}, P_{R 3}^{L, \text { model }}, \ldots, P_{R N}^{L, \text { model }}\right)$ sets that are stored in the database. In total, $N_{L}$ sets of $N$ values of $P_{R i}^{L, \text { model }}$ are precalculated and stored in a fingerprinting database, i.e., the received power at $N_{L}$ locations from each of the $N$ LEDs, according to the LoS channel model from Section II. For the configuration under test, $N=4$ and $N_{L}=2500^{2}=6250000$, meaning that 25 million values are stored. It is clear that more advanced search algorithms will be useful here, in order to quickly find the location with the lowest $C_{\text {square. }}^{L}$.

\section{REsults}

Figs. 4, 5, 6, and 7 show the cdfs that are obtained from $10^{4}$ simulations at each of the locations A to $\mathrm{G}$ in Fig. 3, for tolerances $T_{L E D}$ of $5,10,15$, and $20 \%$, respectively. Table II lists the median $\left(p_{50}\right)$ and 95\%-percentile $\left(p_{95}\right)$ values of the errors for the seven locations and four tolerance values. For $T_{L E D}=5 \%$, median errors are $2.34 \mathrm{~cm}$ at most $(\mathrm{C})$. Maximal errors are limited to $5.58 \mathrm{~cm}(\mathrm{C})$, except at location $\mathrm{B}$, where the maximal error amounts to $17.61 \mathrm{~cm}$. Fig. 4 indeed shows this remarkable behaviour at location $\mathrm{B}$, where half of the LED configurations lead to significantly larger errors than at the other positions. The reason is that the gradient of the LED power is lower just below a LED, so that a certain deviation of the received power corresponds to a relatively larger distance error when measured just below the LED.

TABLE II: Median $\left(p_{50}\right)$ and maximal $\left(p_{95}\right)$ errors $(\mathrm{cm})$ at each of the seven locations A-G (see Fig. 3) for four tolerance values $T_{L E D}$ of the LEDs.

\begin{tabular}{|c|c|c|c|c|c|c|c|c|}
\hline $\mathbf{T}_{\text {LED }}$ & & $\mathbf{A}$ & B & $\mathrm{C}$ & D & $\mathbf{E}$ & $\mathbf{F}$ & $\mathbf{G}$ \\
\hline \multirow[t]{2}{*}{$5 \%$} & $\overline{~ p_{50}}$ & 1.2 & 1.7 & 2.3 & 1.9 & 1.6 & 1.4 & 2.2 \\
\hline & p95 & 2.4 & 17.5 & 5.6 & 4.1 & 3.5 & 4.4 & 5.2 \\
\hline \multirow[t]{2}{*}{$10 \%$} & $p_{50}$ & 2.3 & 3.3 & 4.7 & 3.8 & 3.3 & 2.7 & 4.4 \\
\hline & P95 & 4.7 & 26.7 & 10.9 & 8.2 & 6.9 & 20.3 & 10.4 \\
\hline \multirow[t]{2}{*}{$15 \%$} & p50 & 3.4 & 4.5 & 7.1 & 5.7 & 5.0 & 4.7 & 6.7 \\
\hline & p95 & 7.1 & 33.8 & 15.8 & 12.7 & 10.5 & 30.0 & 15.6 \\
\hline \multirow[t]{2}{*}{$20 \%$} & P50 & 4.6 & 5.6 & 9.5 & 7.6 & 6.5 & 6.6 & 9.0 \\
\hline & P95 & 9.6 & 40.1 & 18.7 & 16.6 & 13.8 & 37.5 & 20.6 \\
\hline
\end{tabular}

All median errors scale more or less linearly with the tolerance values: the errors increase by approximately 100 , 200 , and $300 \%$, when $T_{L E D}$ increases from $5 \%$ to 10,15 , and $20 \%$, respectively. For these positions, median errors $\left(p_{50}\right)$ vary between 2 and $5 \mathrm{~cm}$ for $T_{L E D}=10 \%, 3$ to $7.5 \mathrm{~cm}$ for $T_{L E D}=15 \%$, and between 4 and $10 \mathrm{~cm}$ for $T_{L E D}=20 \%$. Apart from positions B and F, maximal errors $\left(p_{95}\right)$ also show a more or less linear increase: values from 2.5 to $5 \mathrm{~cm}$ for $T_{L E D}=5 \%$, from 5 to $10 \mathrm{~cm}$ for $T_{L E D}=10 \%, 7$ to $15 \mathrm{~cm}$ for $T_{L E D}=15 \%$, and between 10 and $20 \mathrm{~cm}$ for $T_{L E D}=$ $20 \%$ are observed. Moreover, $p_{95}$ values are between 2 and 2.5 times as high as the median errors for all values of $T_{L E D}$. As mentioned earlier, maximal errors at location $\mathrm{B}$ are at least three times as high as at other locations for $T_{L E D}=$ $5 \%$. Although this error does not increase at the same rate as for the other locations when $T_{L E D}$ increases, $p_{95}$ is still at least twice as high as at the other locations for $T_{L E D}=$ $20 \%$. Maximal errors at location $\mathrm{F}$ are in line with the other locations for $T_{L E D}=5 \%$, but for higher $T_{L E D}$ values $(\geq$ $10 \%$ ), a trend similar to that at location B is observed: a large portion of the LED configurations leads to remarkably higher errors compared to locations A-C-D-E-G (see Figs. 5, 6, and 7 


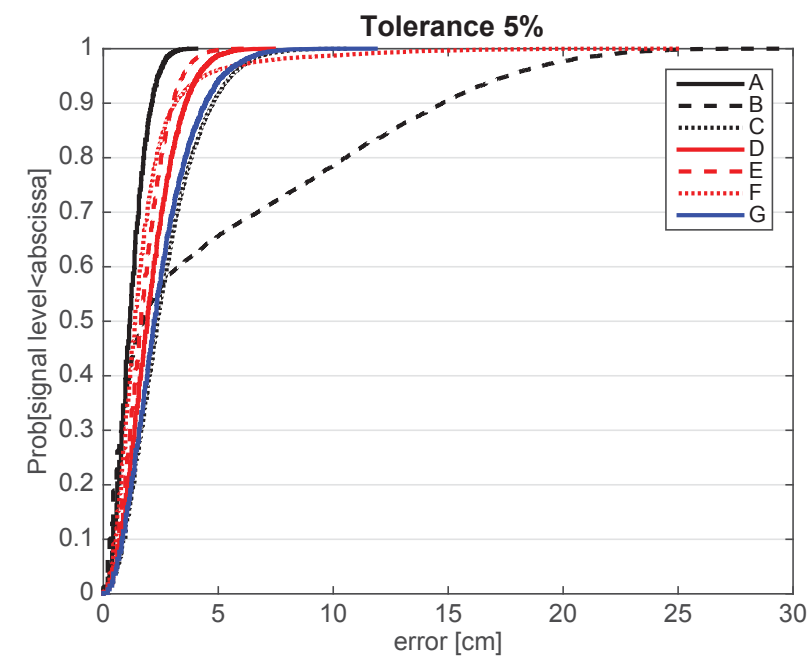

Fig. 4: Cdfs of the errors obtained from 10000 simulations at the locations $A$ to $G$ for a LED tolerance value of $5 \%$.

\section{vs. Fig. 4).}

In general, position A (middle of the room, middle between the four LEDs) shows the lowest median $(<5 \mathrm{~cm})$ and maximal $(<10 \mathrm{~cm})$ errors.

Fig. 8 collects for positions A, B, and F the cdfs of the errors for the four values of $T_{L E D}$, where the $\mathrm{x}$-axis has been limited to $40 \mathrm{~cm}$ for reasons of clarity. It shows that for position $\mathrm{A}$ (black curves), the errors increase in a regular way as $T_{L E D}$ increases. The same counts for position $F$ (blue curves), but the increase of the errors is significantly larger. Finally, for position B (red curves), it can be seen that the smallest $40 \%$ of the errors is affected in a very limited way as $T_{L E D}$ increases, and that these errors are lower than at positions $\mathrm{A}$ and $\mathrm{F}$. However, the largest half of the errors at B is (significantly) larger than at $\mathrm{A}$ (and $\mathrm{F}$ ).

In order to gain more insight into the position-dependency of the error, the errors for $T_{L E D}=10 \%$ within the top right quarter of Fig. 3 (square formed by sides $\mathrm{AE}$ and $\mathrm{CE}$ ) have been simulated on a denser grid of 50 by 50 positions, but due to calculation time, for only 100 instead of 10000 simulations per position. Since the error distribution should be symmetric around line A-C, the 100 errors per position have additionally been mirrored around this symmetry line, so that a symmetrical pattern was obtained with 200 simulations per position. Figs. 9 and 10 show the resulting spatial error map of the median $\left(p_{50}\right)$ and maximal $\left(p_{95}\right)$ error respectively. The axis coordinates adhere to the system used in Table I, and the white dots indicate positions A-G. Position B at $(1.25 ; 1.25)$ is not indicated to better visualize the rapid variation of the error at that location. The median error over the entire area equals $4.21 \mathrm{~cm}$, the maximal error $10.75 \mathrm{~cm}$.

Fig. 9 shows that the largest median errors are found under the LED ( 7 to $9 \mathrm{~cm})$, but at the exact location under the LED (position B), the error remains limited to $3.35 \mathrm{~cm}$ (see Table II and the blueish spot in the middle of the red area in Fig. 9).

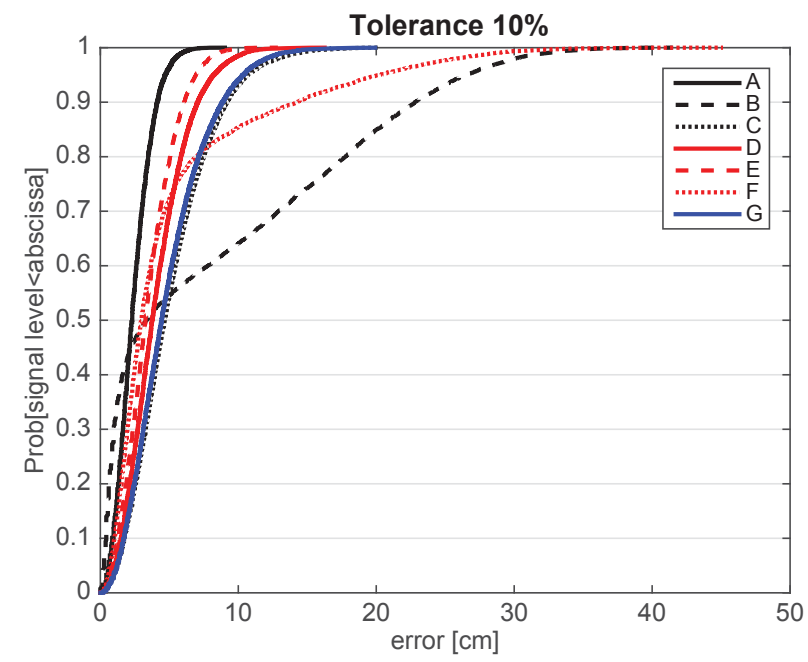

Fig. 5: Cdfs of the errors obtained from 10000 simulations at the locations $\mathrm{A}$ to $\mathrm{G}$ for a LED tolerance value of $10 \%$.

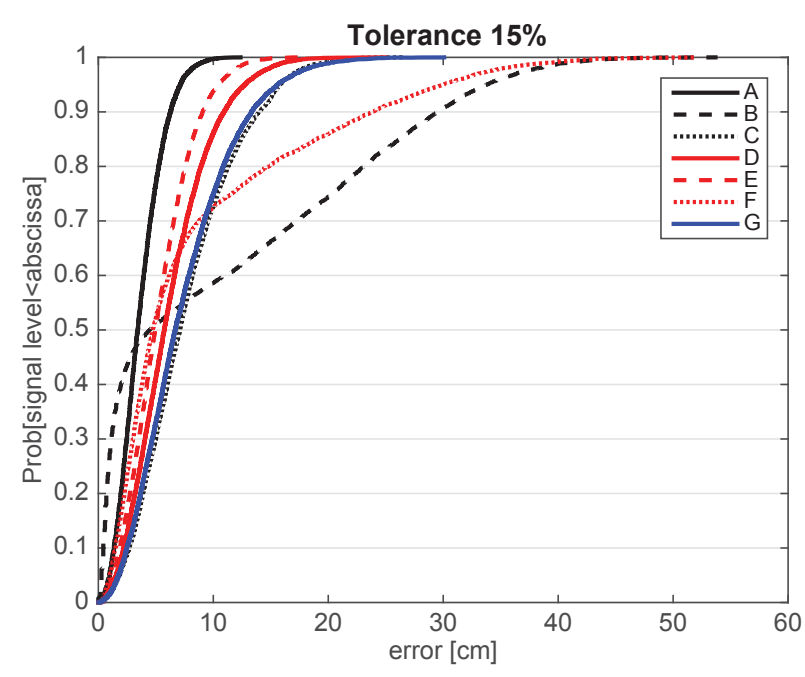

Fig. 6: Cdfs of the errors obtained from 10000 simulations at the locations $A$ to $G$ for a LED tolerance value of $15 \%$.

Further, median errors appear to be larger along the sides of the square that is formed by the four LEDs and towards the corners. Unlike for the median errors, the largest maximal errors in Fig. 10 do occur exactly below the LED $(26.54 \mathrm{~cm}$, see also Table II). With respect to position F, Figs. 9 and 10 show that although larger median errors are observed right of position $\mathrm{F}$ (for $\mathrm{x}$ around $1.5 \mathrm{~m}$, see Fig. 9), maximal errors are large exactly at $\mathrm{F}$ ( $\mathrm{x}$ around $1.25 \mathrm{~m}$, see Fig. 10).

These figures indicate that interesting future work consists of investigating the impact of the Lambertian order, the LED height, and the LED positions on the error distributions. Further, it is to be investigated if similar distributions are obtained when other cost metrics for positioning are used. 


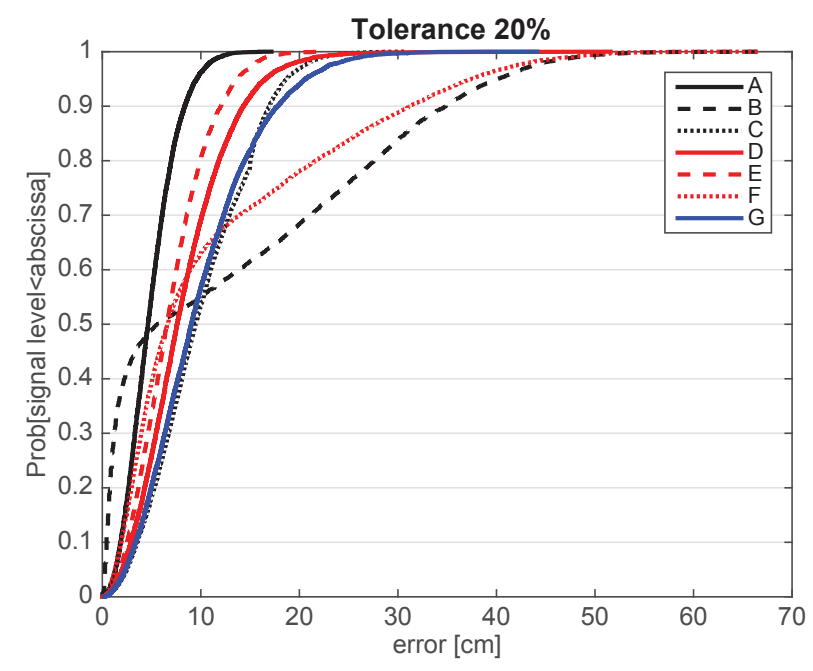

Fig. 7: Cdfs of the errors obtained from 10000 simulations at the locations $A$ to $G$ for a LED tolerance value of $20 \%$.

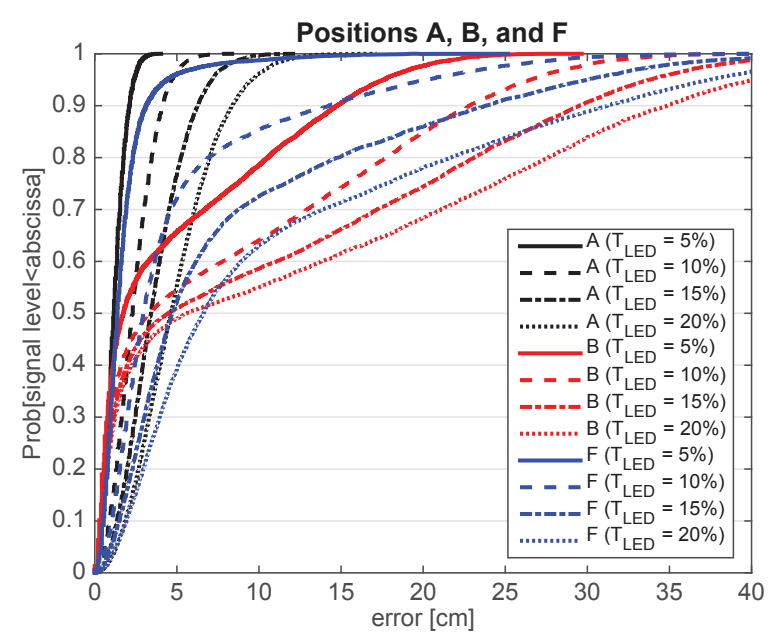

Fig. 8: Cdfs of the errors obtained from 10000 simulations at the locations A, B, and F for all four LED tolerance values.

\section{A. Comparison with impact of noise and reflections}

Fig. 11 shows the median positioning error for each of the seven considered locations, as a function of the standard deviation $\sigma_{N}$ [W] of the observed noise power. It shows that from $\sigma_{N}$ values of around $10^{-7} \mathrm{~W}$ and higher, the median error becomes larger than $1 \mathrm{~cm}$. Location $\mathrm{C}$ (the furthest from the LEDs) is most prone to errors due to noise, while A (at the center of the 4 LEDs) is most resilient to noise. In our lab, a $\sigma_{N}$ of around $1.4 \cdot 10^{-7} \mathrm{~W}$ was obtained. Table III shows the median $\left(p_{50}\right)$ and maximal $\left(p_{95}\right)$ values of the errors for the seven locations, considering LED power uncertainty and an assumed value of $\sigma_{N}=1.4 \cdot 10^{-7} \mathrm{~W}$. The table shows that at locations $\mathrm{A}, \mathrm{E}$, and $\mathrm{G}$ (the locations where noise has the lowest impact according to Fig. 11), the increase of the errors with respect to the errors listed in Table II (i.e.,

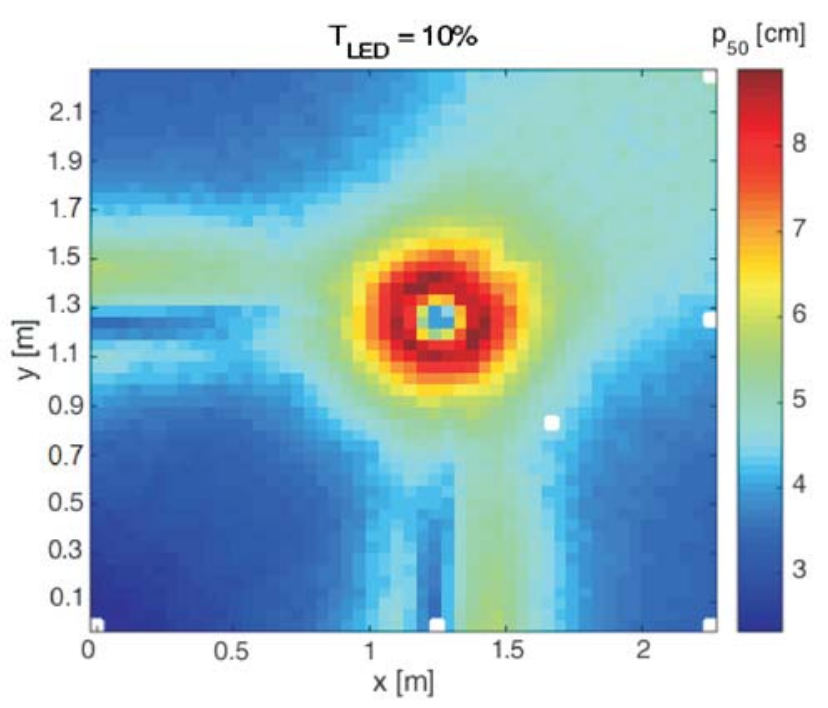

Fig. 9: Spatial distribution of median error $\mathrm{p}_{50}$ obtained from 200 simulations in the square A-C-E of Fig. 3, for a LED tolerance value of $10 \%$.

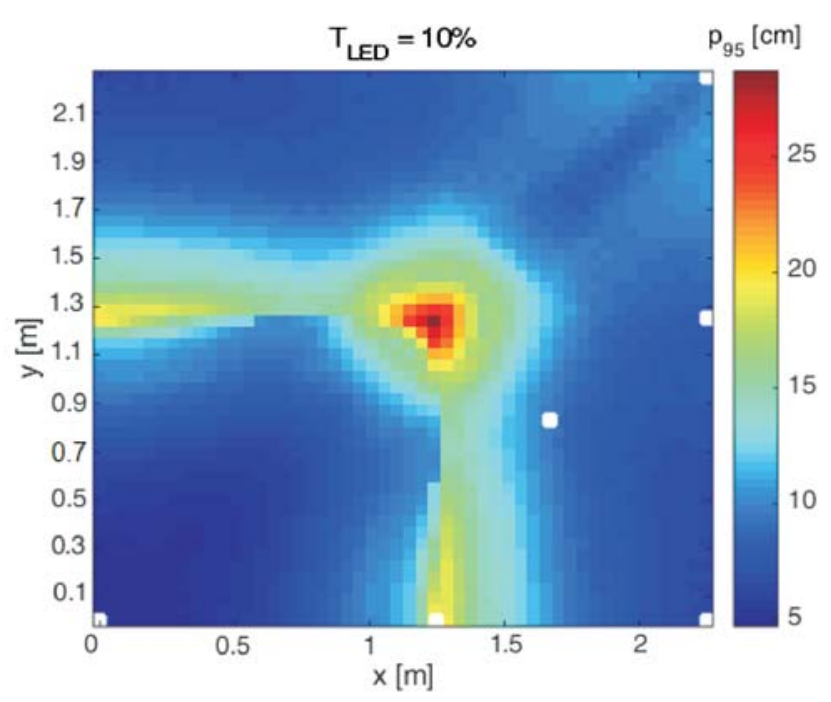

Fig. 10: Spatial distribution of maximal error $\mathrm{p}_{95}$ obtained from 200 simulations in the square A-C-E of Fig. 3, for a LED tolerance value of $10 \%$.

without considering noise) is indeed very limited: average errors increase at most $2 \%$. At locations $\mathrm{B}, \mathrm{D}$, and $\mathrm{F}$, an average error increase between 6 and $10 \%$ is noticed. At location C, Fig. 11 showed that noise had the largest impact on the positioning error. This is confirmed in Table III: errors increase by $51 \%$. On average, the relative impact of adding noise is obviously larger for lower $T_{L E D}$ values. In [9], the impact of reflections on the positioning accuracy was assessed for the same configuration. The median and maximal errors obtained for this configuration due to a LED tolerance of $10 \%$ were equal to 4.21 and $10.75 \mathrm{~cm}$, respectively. Using the same 


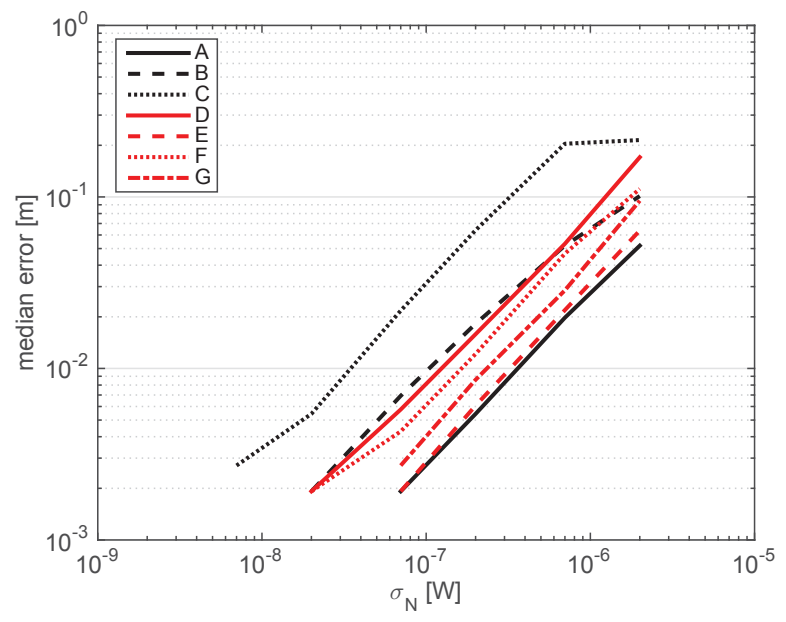

Fig. 11: Impact of noise on positioning accuracy for different positions.

cost metric 'square' [9], the median and maximal errors due to reflections amounted to 8.7 and $11.6 \mathrm{~cm}$ respectively, assuming a wall reflectance factor of 0.3 . It can be concluded that for the given configuration, the impact of noise is smaller than that of the LED power uncertainty, although the noise impact also becomes significant when moving further away from the LEDs. The impact of reflections is more or less comparable to that of a tolerance on the LED power of $10 \%$.

TABLE III: Median $\left(p_{50}\right)$ and maximal $\left(p_{95}\right)$ errors $(\mathrm{cm})$ at each of the seven locations A-G (see Fig. 3) for four tolerance values $T_{L E D}$ of the LEDs and a $\sigma_{N}$ value of $1.4 \cdot 10^{-7} \mathrm{~W}$.

\begin{tabular}{|c|c|c|c|c|c|c|c|c|}
\hline $\mathbf{T}_{\text {LED }}$ & & $\overline{\mathbf{A}}$ & $\bar{B}$ & $\mathrm{C}$ & D & $\mathbf{E}$ & $\mathbf{F}$ & $\mathbf{G}$ \\
\hline \multirow[t]{2}{*}{$5 \%$} & $p_{50}$ & 1.2 & 2.2 & 5.0 & 2.3 & 1.7 & 1.7 & 2.3 \\
\hline & p95 & 2.5 & 17.4 & 13.7 & 5.0 & 3.5 & 6.0 & 5.3 \\
\hline \multirow[t]{2}{*}{$10 \%$} & p50 & 2.3 & 3.7 & 6.5 & 4.0 & 3.3 & 3.2 & 4.6 \\
\hline & p95 & 4.9 & 26.9 & 16.7 & 8.8 & 7.0 & 20.6 & 10.6 \\
\hline \multirow[t]{2}{*}{$15 \%$} & p50 & 3.5 & 4.9 & 8.4 & 5.9 & 4.9 & 4.9 & 6.8 \\
\hline & p95 & 7.2 & 33.4 & 18.9 & 12.9 & 10.4 & 29.8 & 15.7 \\
\hline \multirow[t]{2}{*}{$20 \%$} & $p_{50}$ & 4.6 & 5.8 & 10.5 & 7.7 & 6.5 & 6.7 & 9.0 \\
\hline & p95 & 9.6 & 39.4 & 20.5 & 16.7 & 13.8 & 37.2 & 20.4 \\
\hline
\end{tabular}

\section{CONCLUSIONS}

In this paper, it is investigated to what extent the uncertainty or tolerance on the actual emitted LED power impacts the RSS-based VLP accuracies. For a $5 \mathrm{~m}$ x $5 \mathrm{~m}$ room with four LEDs, cdfs of the resulting positioning errors are constructed, based on a Monte Carlo simulation consisting of $10^{4}$ simulations. At most locations, median errors are below $2.5 \mathrm{~cm}$ for a tolerance of $5 \%$, and these errors increase approximately linearly as the tolerance values on the LED powers increase, with most median errors below $10 \mathrm{~cm}$ for a tolerance of $20 \%$. However, it is shown that median and maximal errors show significant local variations, where the highest positioning errors are observed at locations just below the LEDs. For tolerance values of only $5 \%$, maximal errors can already be as high as $17 \mathrm{~cm}$ just below the LED, and increase up to $40 \mathrm{~cm}$ for tolerance values of $20 \%$. For the considered configuration, a tolerance value of $10 \%$ has an impact on positioning accuracy that is comparable to that of wall reflections, and has a larger impact than noise. Future work includes an experimental analysis of actual LED power tolerances, and an analysis of different Lambert modes and LED positions. Further, the impact on the error will be investigated when the powers of the different LEDs are correlated, since LEDs from a same batch might be likely to have similar deviations from the tabulated power. Also, the study can be repeated for commercial LED sources instead of Lambertian radiators.

\section{REFERENCES}

[1] A. Jovicic, J. Li, and T. Richardson, "Visible light communication: opportunities, challenges and the path to market," IEEE Communications Magazine, vol. 51, no. 12, pp. 26-32, December 2013.

[2] J. Armstrong, Y. A. Sekercioglu, and A. Neild, "Visible light positioning: A roadmap for international standardization," IEEE Communications Magazine, vol. 51, no. 12, pp. 68-73, December 2013.

[3] D. Dardari, A. Conti, U. Ferner, A. Giorgetti, and M. Z. Win, "Ranging with ultrawide bandwidth signals in multipath environments," Proceedings of the IEEE, vol. 97, no. 2, pp. 404-426, 2009.

[4] J. Trogh, D. Plets, L. Martens, and W. Joseph, "Advanced real-time indoor tracking based on the viterbi algorithm and semantic data," International Journal of Distributed Sensor Networks, vol. 11, no. 10, 2015. [Online]. Available: http://dsn.sagepub.com/content/11/10/271818.abstract

[5] TCI Telecomunicazioni, "CD MAXI JOLLY DALI," Tech. $\quad$ Rep. $\quad$ [Online]. http://www.tcisaronno.com/PDF/istruzioni/DC_MAXI_JOLLY_DALI.pdf

[6] Citiled, "Citiled COB CLU038-1205C4 Datasheet, $\quad$ Tech. Rep. [Online]. Available: http://ce.citizen.co.jp/lighting_led/dl_data/datasheet/en/COB_5/CLU0381205C4_P3537_0216.pdf

[7] T. Komine and M. Nakagawa, "Fundamental analysis for visible-light communication system using led lights," IEEE Trans. Consumer Electronics, vol. 50, pp. 100-107, 2004.

[8] S. D. Lausnay, L. D. Strycker, J.-P. Goemaere, N. Stevens, and B. Nauwelaers, "A Visible Light Positioning system using Frequency Division Multiple Access with square waves," in 2015 9th International Conference on Signal Processing and Communication Systems (ICSPCS), Cairns, QLD, Australia, 14-16 Dec 2015, pp. 1-7.

[9] D. Plets, A. Eryildirim, S. Bastiaens, N. Stevens, L. Martens, and W. Joseph, "A performance comparison of different cost functions for rss-based visible light positioning under the presence of reflections," in Proceedings of the 4th ACM Workshop on Visible Light Communication Systems at the 23rd Annual International Conference on Mobile Computing and Networking. ACM Press, 2017, pp. 37-41. [Online]. Available: http://dx.doi.org/10.1145/3129881.3129888 